Check for updates

Cite this: RSC Adv., 2017, 7, 54100

Received 21st June 2017

Accepted 20th November 2017

DOI: 10.1039/c7ra06913h

rsc.li/rsc-advances

\section{In vitro and in vivo studies of a gelatin/ carboxymethyl chitosan/LAPONITE® composite scaffold for bone tissue engineering}

\author{
$\mathrm{Li}$ Tao, $\uparrow$ Liu Zhonglong, $\uparrow$ Xiao Ming, Yang Zezheng, Liu Zhiyuan, Zhou Xiaojun (DD * \\ and Wang Jinwu (iD *
}

In the present study, we fabricated a biocomposite scaffold composed of carboxymethyl chitosan (CMC), gelatin and LAPONITE® (Lap) nanoparticles via freeze-drying and investigated its potential use in bone tissue engineering. The prepared gelatin/carboxymethyl chitosan (GC) scaffolds and laponiteincorporated scaffolds were characterized by scanning electron microscopy (SEM), and Fourier transform infrared spectroscopy (FTIR) analyses. The swelling and biodegradation were also investigated. In vitro assays such as cell attachment and proliferation, osteogenic differentiation of rat bone marrow-derived mesenchymal stem cells (rBMCSs) grown on those scaffolds and in vivo cranial bone defect assays were further carried out. We found that our prepared scaffolds had a porous architecture, and the increased Lap content resulted in improved mechanical strength, whereas the swelling ratio and degradation rate decreased. In vitro cell proliferation and live cell staining experiments demonstrated that the addition of Lap (5 and $10 \mathrm{wt} \%$ relative to gelatin, GC-Lap5\% and GC-Lap10\% respectively) would facilitate cell proliferation, but caused an inhibition effect at 15\% of Lap content (GC-Lap15\%). Furthermore, GCLap10\% induced a higher degree of osteogenic differentiation of rBMSCs compared with the GC scaffold and GC-Lap5\% scaffold. More importantly, in vivo cranial defect experiments revealed that the addition of Lap into the GC scaffold promoted bone regeneration. These findings indicate that a composite scaffold with Lap incorporation is a promising material for bone tissue engineering.

\section{Introduction}

Bone defects and non-unions in the clinic have traditionally been repaired with autografts or allografts. ${ }^{1}$ However, these approaches are restricted by donor shortages, donor-site morbidity, and the risk of disease transfer. ${ }^{2,3}$ A promising alternative to bone grafting is bone tissue engineering (BTE), in which bone tissue is constructed de novo by combining boneforming cells, growth factors, and three-dimensional (3D) porous scaffolds. ${ }^{4}$

The scaffold used in BTE acts as an artificial extracellular matrix (ECM) to support cell adhesion and promote cell differentiation without hindering proliferation, thereby guiding bone regeneration. ${ }^{5}$ Recently, many types of synthetic and natural polymer materials have been used as scaffolds and have shown promising results for bone regeneration. ${ }^{6-9}$ Gelatin, a hydrolytic derivative of collagen, has been widely used in the

Shanghai Key Laboratory of Orthopaedic Implant, Department of Orthopaedic Surgery, Shanghai Ninth People's Hospital Affiliated to Shanghai Jiao Tong University School of Medicine, Room 701, No. 3 Building, 639 Zhizaoju Road, Shanghai 200011, China. E-mail: jinwuwang123@sina.com; xjz362@163.com; Fax: +86 21 63139920; Tel: +8613301773680

$\dagger$ These authors contributed equally to this work. biomedical field due to its stable physicochemical properties, lack of antigenicity, and low cost. ${ }^{\mathbf{1 0 , 1 1}}$ In addition, the presence of an Arg-Gly-Asp-like sequence in gelatin can also promote cell attachment, differentiation, and proliferation. ${ }^{12,13}$ However, low mechanical strength, poor elasticity, and morphological instability limit its biological applications. ${ }^{\mathbf{1 4}}$ To improve chemical and biological performance, crosslinking or combining gelatin with other biopolymers has been proposed. ${ }^{15}$ Carboxymethyl chitosan (CMC), a water-soluble derivative of chitosan, has high aqueous solubility, controllable biodegradability, and osteogenesis-inducing potential as compared to chitosan. ${ }^{\mathbf{1 6}}$ Previous studies have shown that blending CMC with gelatin can improve the physicochemical and biological properties of the gel, including mechanical strength, biocompatibility, and capacity to induce osteogenesis. ${ }^{\mathbf{1 6 , 1 7}}$ Thus, gelatin/ carboxymethyl-chitosan (GC) composites have various applications in wound healing and cartilage and bone regeneration. ${ }^{15-17}$ Despite interesting physical, chemical, and biological properties, insufficient osteoinductive properties and poor mechanical loading make the GC composites scaffolds far from satisfactory.

Nanoclay, namely synthetic silicate, has a high aspect-ratio morphology that enhances the structural integrity of nanocomposites into which it is incorporated. The physical and 
chemical properties of nanocomposite matrices can be modified by adding nanoclay. ${ }^{18,19}$ For example, the incorporation of montmorillonite into poly(lactic acid) scaffolds increased its compressibility to a level comparable to that of cancellous bone. ${ }^{20}$ Cloisite, another clay, were used to adjust the mechanical parameters of poly[ethylene-co-(vinyl acetate)]. ${ }^{21}$ Halloysite, when added as an inorganic filler via electrospinning was found to improve the mechanical properties of poly( $\varepsilon$-caprolactone) (PCL)/ nanocellulose fibrous scaffolds. ${ }^{22}$ Despite advances in the physical and chemical properties of polymers by reinforcement with nanoclay, few studies have examined the interaction between clay-based scaffolds and cells. ${ }^{23-26}$ Ambre et al. reported that incorporating synthetic silicate into chitosan/polygalacturonic acid scaffolds promoted osteogenic differentiation of human mesenchymal stem cells (hMSCs). ${ }^{26}$ Hong Zhuang et al. found out that montmorillonite-intercalated gelatin/chitosan induced the adhesion and proliferation of rat stromal stem cells; ${ }^{27}$ Ganesh Nitya et al. demonstrated hMSCs seeded on scaffolds embedded with halloysite nanoclay showed higher proliferation rate and alkaline phosphatase (ALP) activity. ${ }^{22}$

LAPONITE $®$ (Lap), a silicate that usually acted as a physical or covalent cross-linker in polymer fabrication; it has recently received much attention because of its excellent physical and chemical properties as well as ideal osteogenesis-inducing potential. ${ }^{23,24,28,29}$ Incorporating Lap into polymers has shown to alter their characteristics such as hydration, dissolution, and mechanical properties. On the other hand, the adhesion, proliferation, and osteogenic potential of cells cultured on these nanocomposites are correlated with Lap concentration. ${ }^{23-25}$ For example, Lap crosslinking not only improved the mechanical strength and network stability of a poly(ethylene oxide) (PEO) nanocomposite polymer, but also enhanced the adhesion, spreading, proliferation, and mineralization of preosteoblast cells. ${ }^{23}$ Recently, an electrospun PCL scaffold was fabricated in which surface roughness, degradation rate, and mechanical properties were markedly altered by adding Lap; moreover, the adhesion, proliferation, and osteogenic differentiation of hMSCs grown on the these scaffolds were dependent on nanoclay concentration. ${ }^{24}$

Despite previous studies have displayed the beneficial effect of Lap in preparing novel bioactive materials in tissue engineering, ${ }^{23,30}$ further in vivo analysis needs to be performed before Lap incorporated materials can be used in clinical applications. Meanwhile, for BTE application, especially in regeneration of large bone defect, silicate nanoparticles developed materials should be highly porous to induce cell migration and allow nutrient transportation as well as to improve bone tissue ingrowth. ${ }^{31}$ In this regard, a promising solution lies in the incorporation of Lap nanoplatelets with polymers to fabricate a 3D porous scaffold. To the best of our knowledge, there have been no studies to date describing the reinforcement of GC scaffolds with Lap for BTE applications. Furthermore, there is no information on the biocompatability and bioactivity of polymer/ Lap composites in vivo. ${ }^{24}$ In the present study, we fabricated a porous Lap-incorporated GC scaffold by freeze drying. The scaffold was characterized with regards to its physical and mechanical properties, biodegradability, and biocompatibility as well as its ability to promote osteogenesis in vitro and in vivo.

\section{Experimental}

\subsection{Materials}

$\alpha$-Minimal Essential Medium ( $\alpha$-MEM), fetal bovine serum, PBS, penicillin, and streptomycin were purchased from Gibco (Carlsbad, CA, USA). Lap $\left(\mathrm{Na}^{0.7+}\left[\mathrm{Mg}_{5.5}: \mathrm{Li}_{0.3} \mathrm{Si}_{8} \mathrm{O}_{20}(\mathrm{OH})_{4}\right]^{0.7-}\right)$ with an average diameter of $25-30 \mathrm{~nm}$ and $1 \mathrm{~nm}$ in thickness was a kindly gift from Donghua University. CMC (degree of deacetylation 96.5\%; MW: 70 000) was purchased from Qingdao Honghai Co. Ltd (Qingdao, China). Gelatin ( $225 \mathrm{~g}$ bloom, type B) was purchased from Sigma-Aldrich (St. Louis, MO, USA). 1-(3Dimethylaminopropyl)-3-ethylcarbodiimide hydrochloride (EDC) and $\mathrm{N}$-hydroxysuccinimide (NHS) were obtained from Energy Chemical (Shanghai, China). Other chemicals were purchased from Sinopharm Chemical Regent Co. Ltd (Shanghai, China). The rBMSCs (passage 3) were purchased from the Type Culture Collection of the Chinese Academy of Sciences, Shanghai, China. The water used in this study was from a Millipore Milli-Q system (Millipore, Bedford, MA) with resistivity of $18.2 \mathrm{M} \Omega \mathrm{cm}$ at $25^{\circ} \mathrm{C}$.

\subsection{Preparation of GC and GC-Lap scaffolds}

A CMC solution was obtained by dissolving $80 \mathrm{mg}$ of powder in ultrapure water at $40{ }^{\circ} \mathrm{C}$. Gelatin powder $(800 \mathrm{mg})$ was added while stirring for $1 \mathrm{~h}$ to obtain the GC solution. For Lap incorporation, appropriate amounts of Lap (5\%, 10\%, and 15\% of the gelatin mass) were introduced into the above mixture. After stirring for $30 \mathrm{~min}$, the mixture was poured into the mold and frozen overnight at $-20{ }^{\circ} \mathrm{C}$, then lyophilized for $48 \mathrm{~h}$. Dried scaffolds were crosslinked using EDC and NHS in a mixed solvent of acetone and water (volume ratio: $4: 1$ ) for $24 \mathrm{~h}$ at $4{ }^{\circ} \mathrm{C}$. The scaffolds were lyophilized and stored at $-20{ }^{\circ} \mathrm{C}$. The synthetic process of GC-Lap scaffolds was illustrated in the Scheme 1.

\subsection{Scaffold characterization}

The surface morphology of prepared scaffolds was examined by SEM (TM-1000; Hitachi, Tokyo, Japan). FTIR spectroscopy was carried out on Nicolet 6700 instrument (Thermo Fisher Scientific, Waltham, MA, USA).

\subsection{Measurement of swelling}

To determine the amount of fluid absorbed, swelling assay was conducted by immersing the scaffolds into PBS ( $\mathrm{pH} 7.4)$ at room temperature. At predetermined time intervals up to $2 \mathrm{~h}$, the swollen samples were taken out from the solution and blotted onto filter paper to remove the excess solution, and the wet weight $\left(W_{\mathrm{w}}\right)$ was recorded. After drying, all the samples were weighted $\left(W_{\mathrm{d}}\right)$. Each sample was carried out in triplicates. The swelling percentage was calculated as follows:

$$
S=\left[\left(W_{\mathrm{w}}-W_{\mathrm{d}}\right) / W_{\mathrm{d}}\right] \times 100 \%
$$



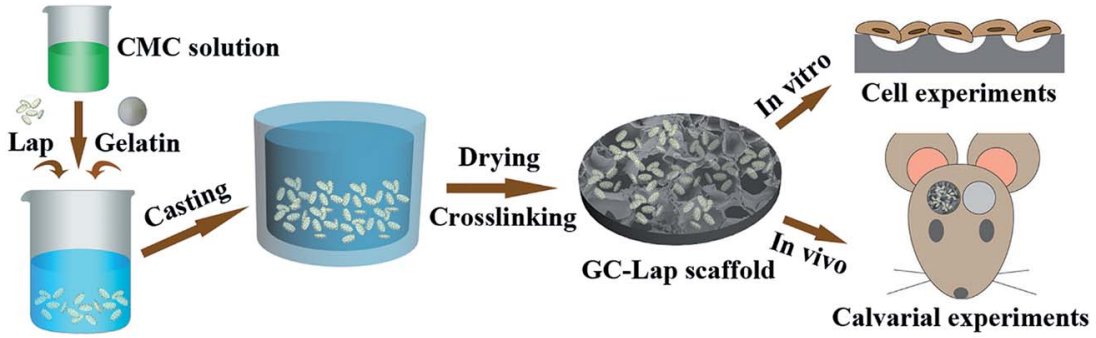

Gelatin/CMC/

Lap mixture

Scheme 1 The schematic diagram of composite scaffold processing.

\subsection{Evaluation of mechanical properties (compression test)}

To assess the mechanical properties of the scaffolds, the compressive properties were measured with a mechanical tester (HY-940FS; Shanghai Hengyu Co., Ltd, Shanghai, China) fitted with a $200 \mathrm{~N}$ load cell and operated at a loading speed of $1 \mathrm{~mm} \mathrm{~min}{ }^{-1}$. Cylindrical samples $(n=5)$ approximately $6 \mathrm{~mm}$ thick and $10 \mathrm{~mm}$ in diameter were compressed until the thickness was reduced to $60 \%$ of the original value. The Young's modulus was determined as the initial linear section of the stress-strain curve.

\subsection{Evaluation of degradation in vitro}

Three specimens of each type of scaffold were immersed in PBS (pH 7.4) containing $8 \times 10^{4} \mathrm{U} \mathrm{ml}^{-1}$ lysozyme at $37^{\circ} \mathrm{C}$, and degradation was assessed by monitoring weight loss. At predetermined time intervals $(1,3,5,7,14$, and 21 days), the scaffolds were removed from the solution, washed with deionized water, freeze-dried under vacuum, and weighed. The degradation ratio was calculated as follows:

$$
D=\left(W_{0}-W_{t}\right) / W_{0} \times 100 \%
$$

where $W_{0}$ and $W_{t}$ represent the original weight and weight after incubation in lysozyme solution up to time $t$ (days), respectively.

\subsection{Cell proliferation assay}

The MTT assay was used to analyze the biocompatibility of the scaffolds (pure GC, GC-Lap5\%, GC-Lap10\%, and GC-Lap15\%). The scaffolds were sterilized by immersion in $75 \%$ ethanol for $12 \mathrm{~h}$ and then rinsed with PBS. Passage 3 rBMSCs $\left(1 \times 10^{4}\right)$ were cultured on the scaffolds for 1,3 , and 7 days. A $20 \mu \mathrm{l}$ volume of MTT solution $\left(5 \mathrm{mg} \mathrm{ml}^{-1}\right)$ was added to each well followed by incubation at $37^{\circ} \mathrm{C}$ for $2 \mathrm{~h} ; 200 \mu \mathrm{l}$ dimethylsulfoxide was then added in the dark on an oscillator to dissolve the formazan crystals. A $100 \mu \mathrm{l}$ aliquot of each sample was transferred to a 96well plate and the optical density (OD) at $492 \mathrm{~nm}$ was measured.

\subsection{Evaluation of cell attachment}

Sterilized scaffolds were placed in a 48 -well plate and equilibrated in culture medium for $1 \mathrm{~h}$. rBMSCs $\left(1 \times 10^{4} /\right.$ well $)$ were seeded on the scaffolds followed by incubation at $37^{\circ} \mathrm{C}$ and $5 \%$ $\mathrm{CO}_{2}$ in a $100 \%$ humidified atmosphere. After 3 days, the culture medium was replaced with $1 \mathrm{ml}$ calcein-AM solution $\left(8 \mu \mathrm{g} \mathrm{ml}{ }^{-1}\right.$ in Hank's balanced salt solution) and the plate was incubated for $15 \mathrm{~min}$ at $37{ }^{\circ} \mathrm{C}$ and $5 \% \mathrm{CO}_{2}$. The morphology of the attached cells was examined by confocal laser scanning microscopy (Leica, Tokyo, Japan).

For SEM analysis, scaffolds were removed from the wells after $12 \mathrm{~h}$ of culture and washed with PBS, then fixed with $2.5 \%$ glutaraldehyde for $2 \mathrm{~h}$. After dehydration in a graded series of ethanol (50\%, 70\%, 80\%, 90\%, and 100\%) and thermostatic drying, the scaffolds were gold sputtered and cell morphologies were analyzed by SEM.

\subsection{ALP staining and quantitative analysis}

Passage 3 rBMSCs were seeded on the surface of the scaffolds (GC, GC-Lap5\%, and GC-Lap10\%) in osteogenic medium in a 24-well plate at a density of $5 \times 10^{4}$ cells per well. On days 7 , 14, and 21, ALP activity was quantified with an ALP detection kit (Jiancheng Technology, Nanjing, China) according to the manufacturer's instructions and normalized to total protein content, which was determined with the bicinchoninic acid assay. For ALP staining, scaffolds cultured for 7 or 14 days were rinsed three times with PBS and then fixed with $4 \%$ paraformaldehyde solution for $10 \mathrm{~min}$. The cells were stained with 5bromo-4-chloro-3-indolylphosphate and nitroblue tetrazolium ALP substrate (Beyotime Institute of Biotechnology, Shanghai, China) for $30 \mathrm{~min}$ at room temperature. The substrate solution was removed and distilled water was added to terminate the reaction.

\subsection{Quantitative real time PCR (qRT-PCR) analysis}

Runx-2, OCN, OPN and ALP expression in rBMSCs was evaluated by qRT-PCR after 7 and 14 days of culture on the scaffolds in osteogenic medium. Total RNA was extracted from cells using TRIzol reagent (Invitrogen), and the concentration and purity were confirmed by measuring OD at 260 and $280 \mathrm{~nm}$. Samples with OD260/280 ratios between 1.9 and 2.1 were used for cDNA synthesis by reverse transcribing $1 \mathrm{mg}$ total RNA in a final volume of $20 \mu \mathrm{l}$ using the PrimeScript RT Reagent kit (Takara Bio, Otsu, Japan). PCR was performed as previously described ${ }^{32}$ using Power SYBR Green PCR Master Mix (Applied Biosystems, Foster City, CA, USA) on a 7500 Real-Time PCR detection system. The primer sequences are shown in Table 1. Relative 
Table 1 Primer sequences used for real-time PCR primer

\begin{tabular}{ll}
\hline Primer & Sequence $\left(5^{\prime}-3^{\prime}\right)$ \\
\hline \multirow{2}{*}{ Runx-2 } & F: $5^{\prime}$-GCGGACGAGGCAAGAGTT-3 \\
& R: $5^{\prime}$-TTGGTGCTGAGTTCAGGGAG-3' \\
OCN & F: $5^{\prime}$-CAGGTGCAAAGCCCAGCGACT-3' \\
& R: $5^{\prime}$-AGGGGATCTGGGTAGGGGGCT-3' \\
OPN & F: $5^{\prime}$-CAGTATCCCGATGCCACA-3' \\
& R: $5^{\prime}$-TTTCCACGCTTGGTTCAT-3' \\
ALP & F: $5^{\prime}$-TCACTTCCGCCCGGAACCCT-3' \\
& R: $5^{\prime}$-TGTCCTGCCGGCCCAAGAGA-3' \\
GAPDH & F: $5^{\prime}$-TGCACCACCAACTGCTTAGC-3' \\
& R: $5^{\prime}$-GGCATGGACTGTGGTCATGAG-3'
\end{tabular}

mRNA levels were determined with the $2^{-\Delta \Delta C_{\mathrm{t}}}$ method, ${ }^{33}$ with glyceraldehydes-3-phosphate dehydrogenase (GAPDH) used as a reference gene. All reactions were carried out in triplicate.

\subsection{Animal experiments}

The use of animals and experimental protocol were approved by the Animal Research Committee of the Ninth People's Hospital, Shanghai Jiao Tong University School of Medicine. The procedure performed was in accordance with the Guide of the Care and Use of Laboratory Animals published by the National Academy of Sciences. The pie-shaped $\left(\varphi 5 \mathrm{~mm} \times 1 \mathrm{~mm}^{3}\right)$ porous scaffolds were sterilized and seeded with rBMSCs. All surgical procedures were performed on 12 week-old male SpragueDawley rats $(n=24)$ as previously described. ${ }^{34}$ A sagittal incision about $2 \mathrm{~cm}$ was made on the scalp. After blunt dissection, the calvarium was exposed. Bilateral critical-sized defects (CSDs) (5 mm-diameter) were created in every rat with an electric trephine (Saeshin, Taegu, South Korea). Then the cellseeded scaffolds were implanted into the defects after $12 \mathrm{~h}$ of culture. The rats were randomly distributed to one of the following groups ( $n=8$ each): (1) blank (right CSD) and GC scaffold with rBMSCs (left CSD); (2) blank (right CSD) and GCLap5\% scaffold with rBMSCs (left CSD); and (3) blank (right CSD) and GC-Lap10\% with rBMSCs (left CSD). Rats showed normal activity after this procedure.

2.11.1 Micro-CT. At 4 and 8 weeks after the surgery, the rats were sacrificed by air embolization. Then the skulls were dissected and fixed in $4 \%$ paraformaldehyde immediately. The morphology of the reconstructed skulls was assessed by microCT (mCT-80; Scanco Medical, Brüttisellen, Switzerland) in highresolution scanning mode (pixel matrix, $1024 \times 1024$; voxel size, $20 \mathrm{~mm}$; slice thickness, $20 \mathrm{~mm}$ ) to determine bone volume. After the scan, an image of the bone was reconstructed using GEHC Micro-View software (GE Healthcare BioSciences, Chalfont-St. Giles, UK). Bone volume/total volume (BV/TV) and bone mineral density were also calculated with the software.

2.11.2 Histological analysis. After micro-CT analysis, the skull of each mouse was dehydrated in increasing concentrations of alcohol (70-100\%) and embedded in polymethyl methacrylate until solidification. Coronal sections (approximately $100 \mu \mathrm{m}$ in thickness) of the central area from each defect were cut and ground. Specimens were polished to a final thickness of approximately $20-30 \mu \mathrm{m}$ and stained with van Gieson's picrofuchsin to identify new bone formation. The area of newly formed bone was quantified with Image Pro Plus software (Media Cybernetics, Rockville, MD, USA) as a percentage of the whole bone defect area.

\subsection{Statistical analysis}

SPSS 17.0 software (SPSS Inc., Chicago, USA) was used for statistical analysis and the data were demonstrated as the means \pm standard deviation (SD). Statistical differences were determined by an analysis of variance (ANOVA) with a level of statistically significance set at $P<0.05$.

\section{Results and discussion}

\subsection{Scaffold characterization}

Open and interconnected pores promote gas and nutrient transport and waste diffusion, which are necessary for cell migration, proliferation, and differentiation. ${ }^{35,36}$ Additionally, an appropriate pore size in scaffolds is critical for promoting vascularization and bone growth. ${ }^{37}$ As observed in Fig. 1, the SEM images showed that our prepared scaffolds in this study were highly porous and interconnected. The pore diameter decreased with the increasing Lap content, ranging from 200$380,160-300,130-250$, and 100-220 $\mu \mathrm{m}$ for pure GC scaffold and GC-Lap scaffolds containing 5\% (GC-Lap5\%), 10\% (GCLap10\%) and 15\% (GC-Lap15\%) Lap, respectively. This suggests that the incorporation of Lap nanoparticles reduced the average pore size in scaffolds, which was to some extent proportional to Lap concentration. It has been reported that a suitable pore size distribution for osteoinductivity or for promoting stem cell infiltration ranges from 100 to $400 \mu \mathrm{m} ;{ }^{38}$ the pore sizes of the prepared GC-Lap scaffolds are within this range. Additionally, with the increase of Lap, the surface roughness in the pore wall of scaffolds increased in a concentration-dependent manner (Fig. 1). This was more evident in the GC scaffolds containing $10 \%$ and 15\% nanoclay (GC-Lap10\% and GC-Lap15\%), showing greater roughness than that of GC-Lap5\%. This is similar to what has been observed in chitosan-based polymer scaffolds containing nanohydroxyapatite, ${ }^{39}$ in which nanoparticles deposited along pore walls and therefore promoted mineralization, protein adsorption, and cell adhesion. ${ }^{\mathbf{4 0}}$

\subsection{Fourier transform infrared (FTIR) spectroscopy analysis}

The FTIR spectra were obtained to confirm the blending of CMC and incorporation of Lap in the Lap-contained GC sample (Fig. 2). In the spectrum of pure gelatin scaffold, typical characteristic absorption bands at 3285 and $3073 \mathrm{~cm}^{-1}$ were attributed to N-H stretching of amide II, while those at 2940, $1631,1544,1231 \mathrm{~cm}^{-1}$ were ascribed to $\mathrm{C}-\mathrm{H}$ stretching of alkyl groups, $\mathrm{C}=\mathrm{O}$ stretching of amide $\mathrm{I}, \mathrm{N}-\mathrm{H}$ stretching of amide II and $\mathrm{N}-\mathrm{H}$ stretching of amide III, respectively. ${ }^{41}$ For CMC, the characteristic absorption bands were observed at 3285, 1585, 1409,1321 and $1054 \mathrm{~cm}^{-1}$ corresponding to the $\mathrm{O}-\mathrm{H}$ stretching vibration, $\mathrm{N}-\mathrm{H}$ stretching vibration of amide II, symmetric stretching vibration of $\mathrm{COO}^{-}$groups, $\mathrm{C}-\mathrm{H}$ stretching vibration 

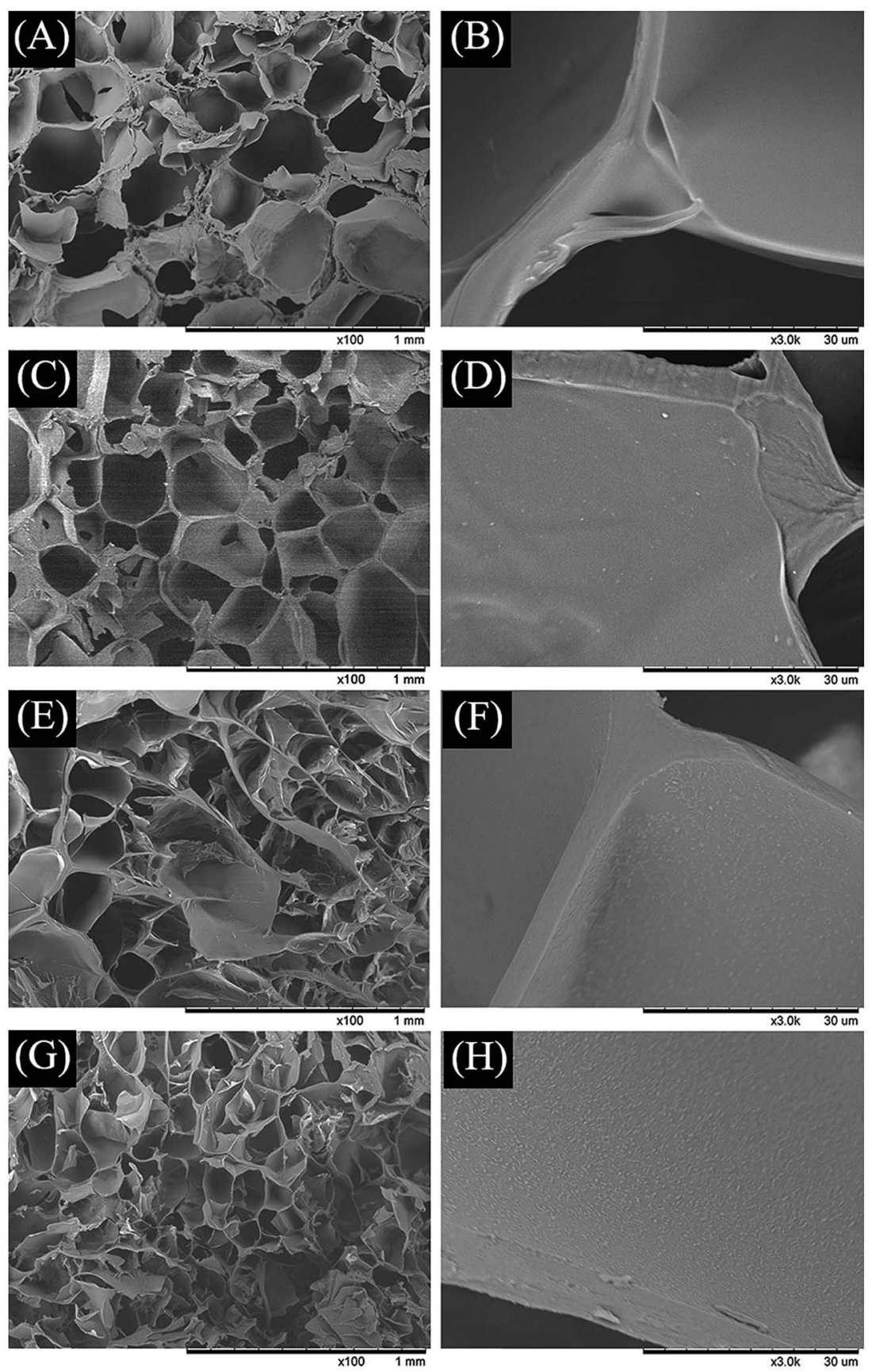

Fig. 1 SEM images of GC (A and B), GC-Lap5\% (C and D), GC-Lap10\% (E and F) and GC-Lap15\% scaffolds (G and H). A, C, E, G show the SEM for magnification $\times 100$; $B, D, F, H$ show the SEM for magnification $\times 3000$.

and $\mathrm{C}-\mathrm{O}$ asymmetric stretching vibration, respectively. ${ }^{\mathbf{1 5 , 4 2}}$ Additionally, the distinctive absorption band located at $992 \mathrm{~cm}^{-1}$ in the spectrum of Lap was assigned to the $\mathrm{Si}-\mathrm{O}$ stretching vibration. ${ }^{43}$ Compared to the spectrum of gelatin samples, the peak at $1403 \mathrm{~cm}^{-1}$ was sharper and the peak intensity at $1077 \mathrm{~cm}^{-1}$ was enhanced in the spectrum of Lapincorporated GC scaffold, which was due to the blending of CMC within the composite. Furthermore, a strong band at $1013 \mathrm{~cm}^{-1}$ was appeared, confirming the incorporation of Lap into the composite.

\subsection{Mechanical testing}

The mechanical strength of scaffolds determines how effectively it can withstand the mechanical load from new tissue growing into the gradually degrading scaffold matrix. ${ }^{\mathbf{4 4}}$ Mechanical properties are also important for cell adhesion, proliferation, and differentiation. ${ }^{23}$ We therefore evaluated the mechanical properties of GC and GC-Lap composite scaffolds in a dry state. As displayed in Fig. 3, both the compressive strength and Young's modulus enhanced with the increased Lap concentration: the compressive strength and Young's modulus of the GC 


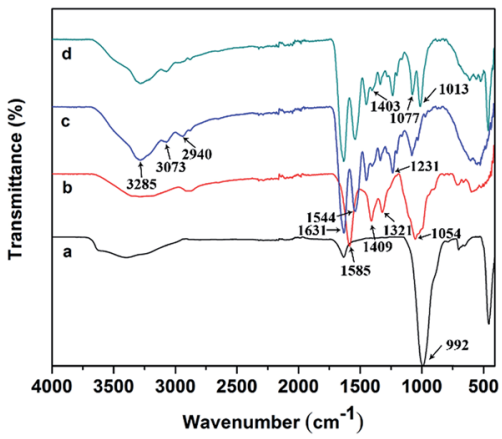

Fig. 2 FTIR spectra of: (a) LAPONITE $®$, (b) CMC, (c) gelatin, (d) GC-Lap scaffold.

scaffold were $0.72 \pm 0.16 \mathrm{MPa}$ and $12.15 \pm 1.29 \mathrm{MPa}$, while the compressive strength and Young's modulus for GC-Lap5\%, GCLap10\% and GC-Lap15\% were $0.80 \pm 0.02 \mathrm{MPa}$ and $14.50 \pm$ $1.17 \mathrm{MPa}, 0.77 \pm 0.02 \mathrm{MPa}$ and $16.25 \pm 0.80 \mathrm{MPa}, 1.01 \pm$ $0.02 \mathrm{MPa}$ and $18.5 \pm 0.82 \mathrm{MPa}$, respectively. This increase in mechanical strength was likely due to the increased density of crosslinks (electrostatic interaction and hydrogen bonds interaction as LAPONITE ${ }^{\circledR}$ worked as noncovalent cross-linkers) between the polymer and Lap and consequent decrease in pore diameter, which yielded a structure that can better withstand stress. ${ }^{\mathbf{3 0 , 4 5}}$ Similar results were reported in other studies where adding silicate nanoparticles to polymer significantly improved the mechanical strength of the nanocomposite films. ${ }^{\mathbf{4 6 , 4 7}}$ It should be noted that despite the compressive strength of the prepared scaffolds didn't reach the standard of cancellous bone (2-20 MPa), ${ }^{48,49}$ the fabricated GC-Lap scaffolds can still be applied in non-load bearing bone defect repair. Moreover, once implanted in vivo, the compressive strength of the porous scaffolds increased with the ingrowth of new bone tissue.

\subsection{Swelling properties of the scaffold}

Swelling capacity is an important property of scaffolds as it affects the exchange of cell nutrients and metabolites. It also increases pore diameter and total porosity, thus providing much larger internal surface area of scaffolds for cell in-growth

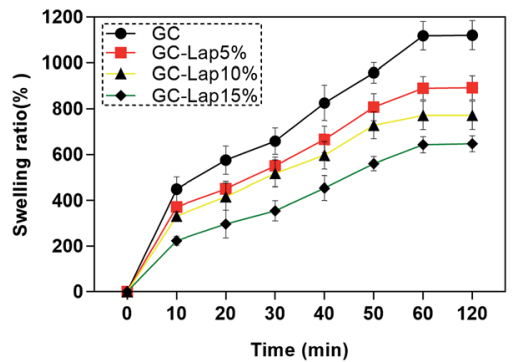

Fig. 4 Swelling behaviour of four different scaffolds as a function of time.

and attachment. However, swelling under physiological conditions must be controlled, as it could cause weakening and rapid degradation of the bone scaffold. ${ }^{38}$ The degree of swelling of the prepared scaffolds was directly proportional to Lap concentration, as scaffolds containing larger amounts of silicate had a higher degree of hydration (Fig. 4). The point of saturated swelling differed among the prepared scaffolds. The sizes of pure GC, GC-Lap5\%, GC-Lap10\%, and GC-Lap15\% scaffolds increased by $449 \%, 372 \%, 332 \%$, and $224 \%$, respectively, after immersion in phosphate-buffered saline (PBS) for $10 \mathrm{~min}$. The swelling ratio increased with immersion time: after $60 \mathrm{~min}$, the for pure GC, GC-Lap5\%, GC-Lap10\%, and GC-Lap15\% scaffolds were $1120 \%, 892 \%, 771 \%$, and $647 \%$, respectively, of their original sizes. At this point, the scaffolds were saturated, as no further swelling was noted with longer incubation times. This is similar to the findings of other studies that the presence of Lap or other nanocomposites in a polymer scaffold decreased the swelling ratio. ${ }^{50}$ This may be due to ionic interactions between silicate and polymer as well as surrounding water molecules that promote the structural stability of the nanocomposite network upon immersion in PBS. The favorable hydrophilic properties of GC-Lap scaffolds facilitate the diffusion of nutrients and metabolites into newly grown tissue. ${ }^{45}$

\subsection{In vitro biodegradation}

Scaffold materials should be biodegradable and bioresorbable at an appropriate rate to match the speed of new bone tissue formation. The biodegradation rate that matches the new bone
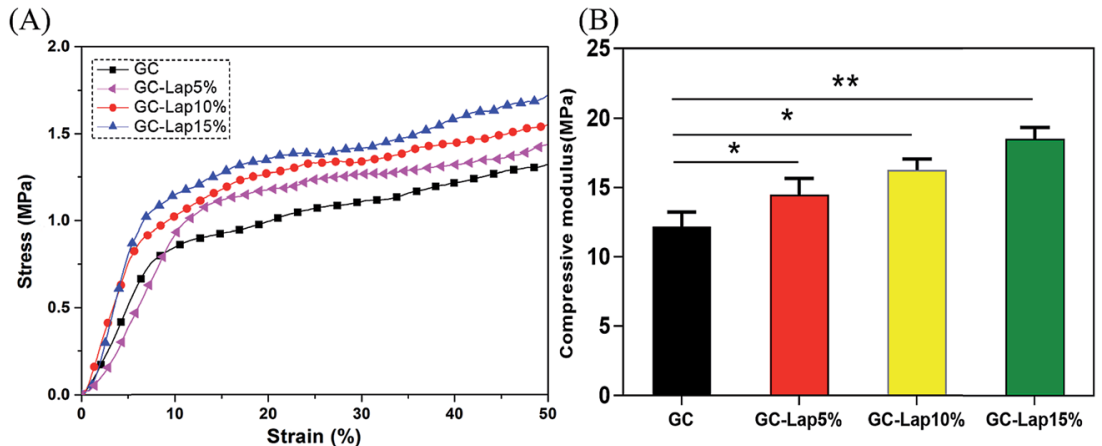

Fig. 3 Mechanical properties of four different scaffolds: (A) strain-stress curves and (B) compressive modulus. * $p<0.05$ compared with the GC. $* * p<0.01$ compared with the GC. 
tissue regeneration rate is an important aspect of scaffold design. ${ }^{51}$ We therefore evaluated the degradation of pure GC and GC-Lap scaffolds immersed in PBS solution with lysozyme addition. The degradation rate of composite scaffold decreased as the added Lap increased (Fig. 5): the rates were 54\%, 45\%, and $26 \%$ for GC-Lap5\%, GC-Lap10\%, and GC-Lap15\% scaffolds, respectively, after incubation for 21 days in lysozyme. However, the pure GC scaffold showed considerable degradation, with only $38 \%$ remaining on day 21 . This is likely due to increased hydrogen bonding and ionic interaction between Lap and water molecules and decreased interaction between polymeric macromolecules and water. ${ }^{24}$ Given that Lap crosslinks enhance the integrity of the GC network; thus, the degradation rate of the scaffold can be regulated by modifying Lap concentration. The slow degradation rate of the scaffold can also provide adequate time for tissue regeneration. ${ }^{45}$

\subsection{In vitro biocompatibility}

Rat bone marrow-derived mesenchymal cells (rBMSCs) are one of the most frequently used cells for investigating tissue repair and reconstruction owing to its multipotency and capacity for self-renewal. ${ }^{52}$ The in vitro biocompatibility of GC-Lap scaffolds was investigated using rBMSCs (passage 3). The proliferation of cells co-cultured with various scaffolds was assessed with the 3 [4,5-dimethylthiazol-2-yl]-2,5-diphenyltetrazolium bromide (MTT) assay. Cell proliferation rates of cells grown on pure GC, GC-Lap5\%, and GC-Lap10\% scaffolds increased with time from 1 to 7 days (Fig. 6). The Lap-incorporated scaffolds stimulated cell growth to a greater extent than pure GC, with GC-Lap10\% being more effective than GC-Lap5\%, especially after 3 days incubation $(P<0.05)$. This may be due to the suitable concentration of dissolution products of Lap $\left[\mathrm{Na}^{+}\right.$, orthosilicic acid $\left(\mathrm{Si}(\mathrm{OH})_{4}\right), \mathrm{Mg}^{2+}$, and $\left.\mathrm{Li}^{+}\right]$, which are similar to those of bioactive glass, and are known to promote cell proliferation. ${ }^{53,54}$ In contrast, in the presence of GC-Lap15\%, cell proliferation was inhibited, possibly due to the adverse effects of high concentrations of Lap. In our study, the optimal Lap concentration for rBMSC proliferation was $10 \%$. A previous study reported that the optimum concentration of cloisite clay in poly[ethylene-co(vinyl acetate)]/cloisite scaffolds for human dermal fibroblast proliferation was $10 \%$, with cell growth inhibited at higher concentrations. ${ }^{21}$ Other studies have found that the ideal Lap

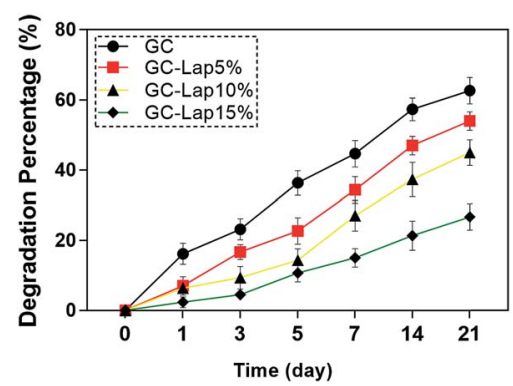

Fig. 5 In vitro biodegradation of porous scaffolds: GC, GC-Lap5\%, GC-Lap10\% and GC-Lap15\%.

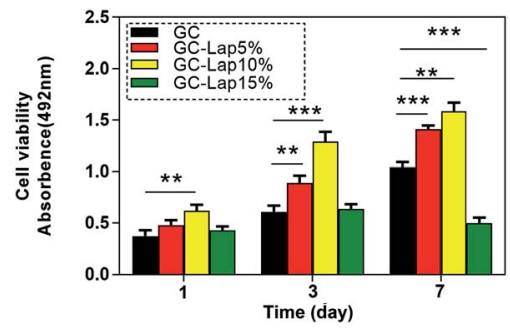

Fig. 6 The proliferation of rBMSCs after 1, 3, and 7 days of culture on the different scaffolds determined by MTT assay. ${ }^{*} p<0.05$ compared with the GC. $* * p<0.01$ compared with the GC. $* * * p<0.001$ compared with the GC.

concentration for osteogenesis is $100 \mu \mathrm{g} \mathrm{ml}{ }^{-1}$, above which cell proliferation is suppressed..$^{28,29}$

\subsection{Cell attachment to scaffolds}

A major consideration in fabricating a scaffold for BTE is that it should support cell adhesion and spreading, since these play a critical role in promoting cell proliferation, differentiation, and formation of the ECM. ${ }^{19}$ To assess the potential of Lap incorporated nanocomposites for BTE applications, the attachment and morphology of rBMSCs on the scaffolds were evaluated by SEM (Fig. 7) and confocal microscopy (Fig. 8). As demonstrated by SEM (Fig. 7), cells on the GC, GC-Lap5\%, and GC-Lap10\% scaffolds had filopodial extensions that increased in number which consistent with Lap concentration after 12 hours of incubation. In contrast, cells on the GC-Lap15\% scaffold were relatively round and extended few filopodia, suggesting that adhesion and spreading were inhibited. The confocal analysis (Fig. 8) revealed a large number of uniformly

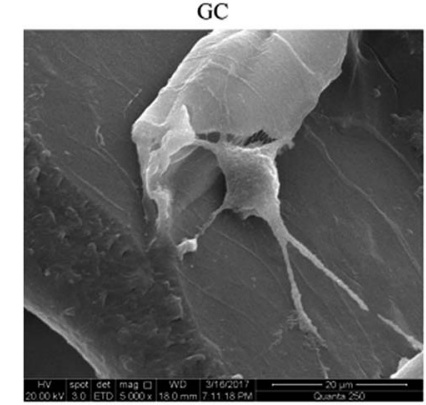

GC-Lap 10\%

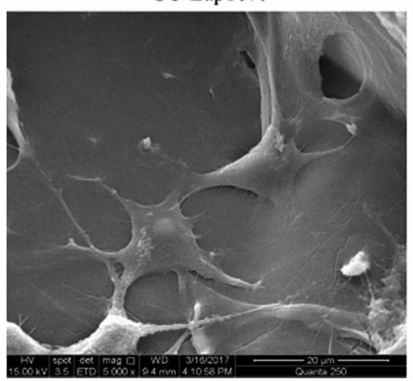

Fig. 7 SEM micrographs of rBMSCs growing on pure GC, GC-Lap5\%, GC-Lap10\% and GC-Lap15\% scaffolds, after $12 \mathrm{~h}$ of culture. 

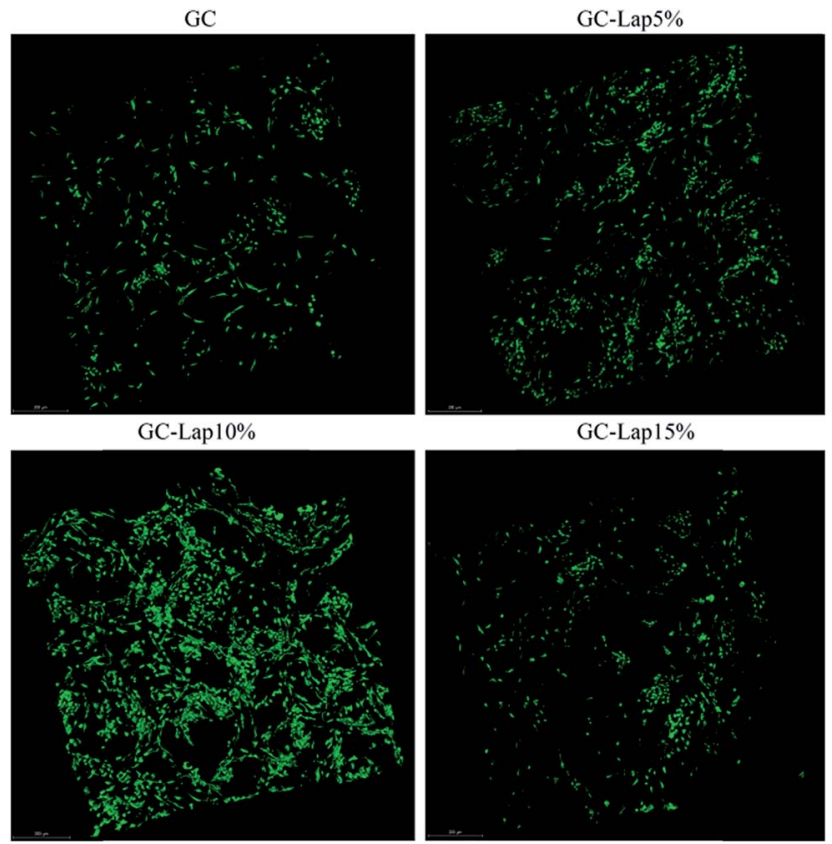

Fig. 8 Confocal images of rBMSCs on pure GC, GC-Lap5\%, GCLap10\% and GC-Lap15\% after 3 days of culture.

distributed cells on GC-Lap5\%, and GC-Lap10\% scaffold surfaces after 5 days of incubation. However, there were fewer cells on the GC and GC-Lap15\% scaffolds. The greater adhesion and spreading in the GC-Lap5\% and GC-Lap10\% groups relative to the GC group may be explained by the existence of a magnetic field generated by adsorption of iron onto Lap. ${ }^{21}$ Additionally, divalent cations such as $\mathrm{Mg}^{2+}$ (a degradation product of Lap) play an important role in the adhesion of cells to biomaterial surfaces via interactions with integrin family proteins. $^{55,56}$ Thus, GC-Lap scaffolds may stimulate rBMSC spreading and adhesion and consequently promote their proliferation. This is in agreement with earlier findings that nanoparticle-containing scaffolds ${ }^{23,56}$ enhanced cell adhesion, which in turn stimulated proliferation. Since GC-Lap15\% inhibited rBMSC proliferation, adhesion, and spreading, it was excluded from subsequent analyses.

\subsection{ALP activity}

Bioactive materials influence cell behavior by releasing soluble components. At $\mathrm{pH}$ values between 7.3 and 8.4, Lap degrades into non-toxic products $\left[\mathrm{Na}^{+}, \mathrm{Si}(\mathrm{OH})_{4}, \mathrm{Mg}^{2+}, \mathrm{Li}^{+}\right] .{ }^{57}$ These dissolution products have been reported to promote collagen type I synthesis and osteoblastic differentiation in vitro. ${ }^{58}$ Based on these observations, we speculated that freeze-dried GC scaffold containing Lap would promote osteogenic differentiation of rBMSCs by providing ionic dissolution products. The osteogenic differentiation potential of passage 3 rBMSCs was evaluated by monitoring ALP activity over a period of 21 days. ALP activity is an early osteogenic differentiation marker that initially increases before decreasing as mineralization of the ECM proceeds. $^{24}$ A greater intensity of ALP staining was observed in rBMSCs grown on GC-Lap10\% as compared to the GC-Lap5\% and pure GC scaffolds after 7 days of culture (Fig. 9A). The intensity of ALP staining in GC-Lap5\% and GCLap10\% further increased on day 14 and was most apparent in GC-Lap10\%. A quantitative analysis of ALP activity on days 7, 14, and 21 revealed an increase on days 7 and 14 in Lapincorporated scaffolds as compared to pure GC scaffold, although a decrease was observed on day 21 (Fig. 9B). In contrast, the GC group showed delayed and moderate activity that peaked on day 14 before decreasing. These results indicate that Lap in polymeric scaffolds induces and promotes osteogenic differentiation of rBMSCs.

\subsection{Osteogenic marker expression in rBMSCs grown on scaffolds}

To further investigate the osteogenic differentiation of rBMSCs induced by GC-Lap scaffolds, we examined the expression of Runt-related transcription factor 2 (Runx-2), osteocalcin (OCN), osteopontin (OPN), and ALP in rBMSCs grown on the scaffolds in osteogenic medium by quantitative real-time PCR (qRT-PCR). Runx-2 is an early marker of osteoblast differentiation that regulates the expression of osteoblast-specific genes such as ALP, collagen-I, and OCN.$^{59}$ The presence of Lap in the scaffolds resulted in higher Runx-2 expression relative to the pure GC scaffold on day 7 , with the highest levels observed in cells grown on GC-Lap10\% (Fig. 10A). OCN, as a non-collagenous protein produced by osteoblasts that signals the termination of osteogenic differentiation, is used to assess bone cell lineage and new bone formation. ${ }^{60}$ There was no significant difference in OCN expression between pure GC and GC-Lap on day 7, but the level was higher in cells cultured on GC-Lap than on the GC scaffold on day 14, especially true for the GC-Lap $10 \%$ group. OPN, another late marker of osteoblast differentiation, showed the
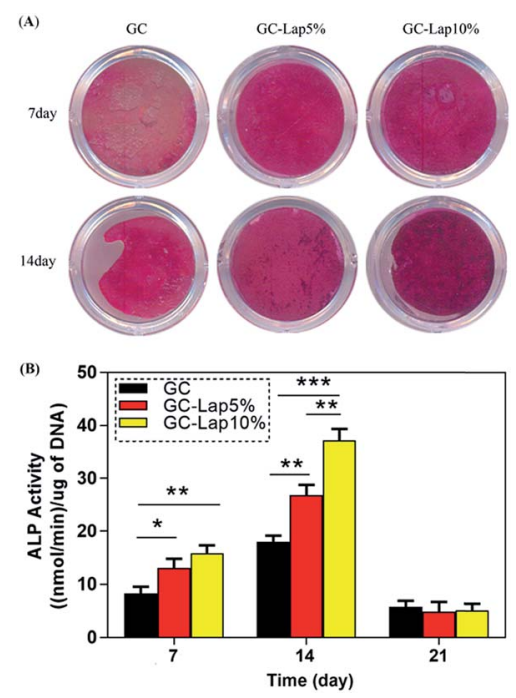

Fig. 9 The osteogenic differentiation of rBMSCs on four different scaffolds. (A) ALP staining at day 7 and day 14. (B) ALP quantification at day 7 , day 14 and day $21 . * p<0.05$ compared with the GC. $* * p<0.01$ compared with the GC. $* * * p<0.001$ compared with the GC. 

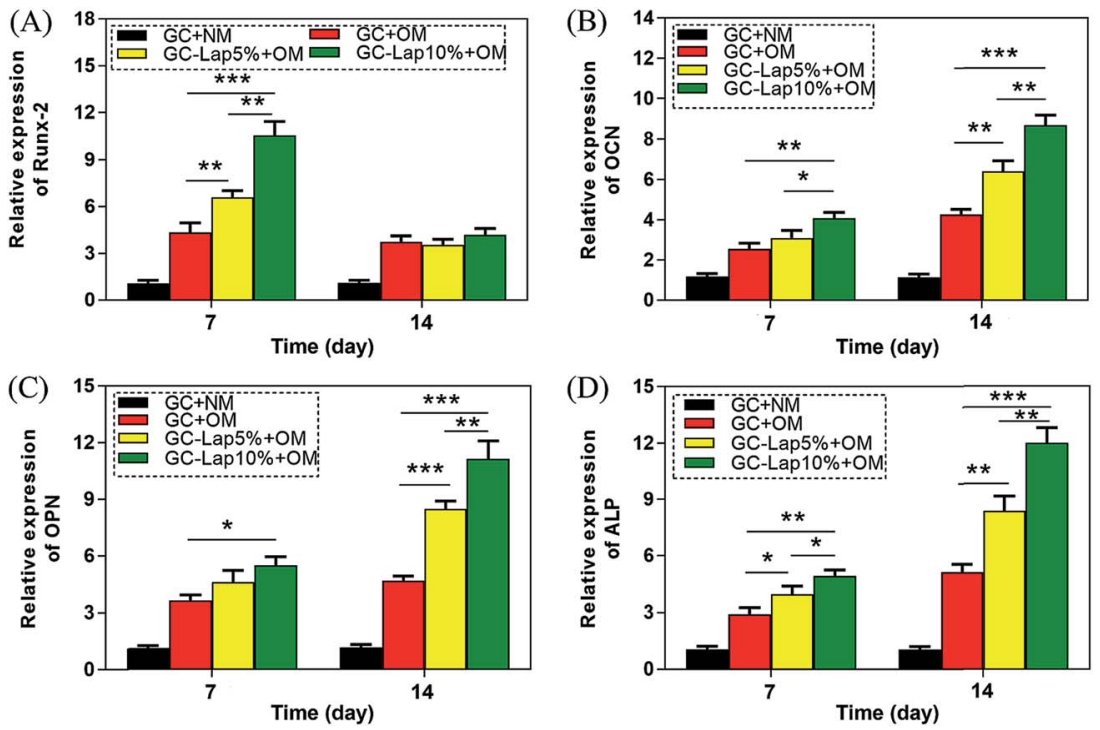

Fig. 10 mRNA expression of osteoblast differentiation marker genes on four different scaffolds (A: Runx-2; B: OCN; C: OPN; D: ALP) at day 7 and day 14. ${ }^{*} p<0.05$ compared with the GC. ${ }^{* *} p<0.01$ compared with the GC. ${ }^{* * *} p<0.001$ compared with the GC.

same trend as OCN, with maximal expression observed in cells grown on GC-Lap $10 \%$ on day 14 . As an early differentiation marker, ALP plays a major role in the conversion of organic pyrophosphate to inorganic phosphate. ${ }^{61}$ As showed in Fig. 10D, ALP was significantly upregulated in cells cultured on the both Lap-containing scaffolds on day 7 relative to the GC group. A similar trend was observed on day 14 , especially for cells grown on GC-Lap10\%. Thus, Lap-containing nanocomposites can effectively promote the osteogenic differentiation of rBMSCs. These results are in agreement with previous studies demonstrating that adding bioactive nanoparticles (silica and hydroxyapatite) to polymeric matrix increased the expression levels of osteogenic differentiation genes..$^{62,63}$

\subsection{Induction of osteogenesis in vivo by GC-Lap scaffolds}

The osteogenesis-inducing capacity of Lap-enriched polymers has not been investigated in vivo. We addressed this by

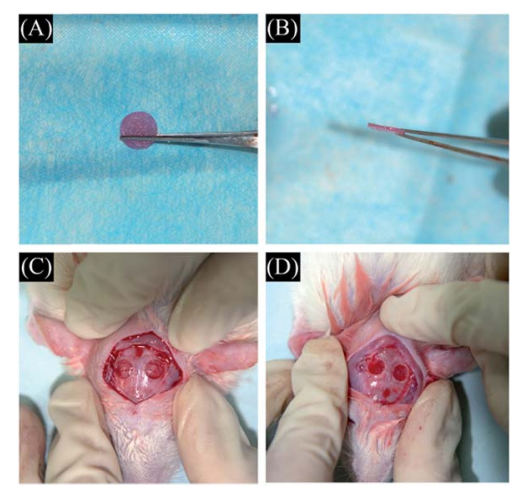

Fig. 11 The scaffolds cut into sections $5 \mathrm{~mm}$ in diameter and $1 \mathrm{~mm}$ thick (A and B), and implanted in bilateral $5 \mathrm{~mm}$ critical-sized calvarial defects $(C$ and $D)$ in rats. implanting an rBMSC-loaded GC-Lap10\% scaffold into a bilateral $5 \mathrm{~mm}$ calvarial bone defects in rats (Fig. 11), with GC and GC-Lap5\% scaffolds serving as controls. Osteogenesis was evaluated by micro-computed tomography (micro-CT) and histology was analyzed by van Gieson's picrofuchsin staining. At 4 weeks after implantation, micro-CT analysis revealed no evidence of new bone formation in the GC group and only a small area of new bone in the GC-Lap5\% group. In contrast, bone regeneration was clearly observed in the GC-Lap $10 \%$ group (Fig. 12). The difference between groups was more pronounced at 8 weeks: the new bone volume to tissue volume ratio (BV/TV) was higher in the GC-Lap10\% group (4 weeks: $10.87 \% \pm 2.20 \%$; 8 weeks: $34.80 \% \pm 2.53 \%)$ than in the GC $(4$ weeks: $2.03 \% \pm 0.76 \%$; 8 weeks: $4.60 \% \pm 1.50 \%$ ) and GC-Lap5\% ( 4 weeks: $5.70 \% \pm 1.38 \%$ and 8 weeks: $18.64 \% \pm 2.18 \%$ ) groups. Interestingly, in Lap-incorporated scaffolds, the new bone generated not only along the defect margin, but also in the central part of the calvarial bone defect. A plausible explanation is chemoattraction by silicate, which is known to promote early angiogenesis, enhance BMSC migration, and promote bone regeneration in critical-sized calvarial defects in mice. ${ }^{64}$
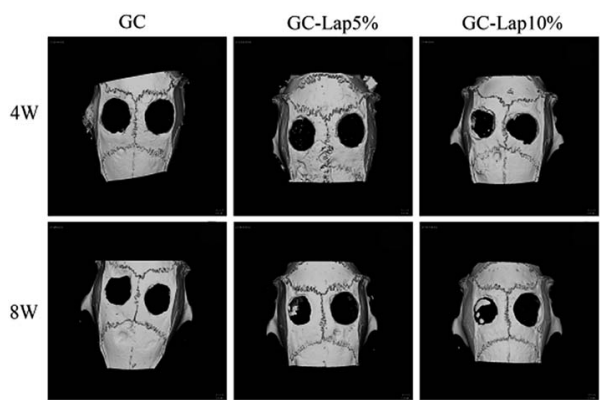

Fig. 12 Virtual micro-CT images from rats receiving the pure GC, GCLap $5 \%$, and GC-Lap10\% scaffolds at 4 and 8 weeks after implantation. 


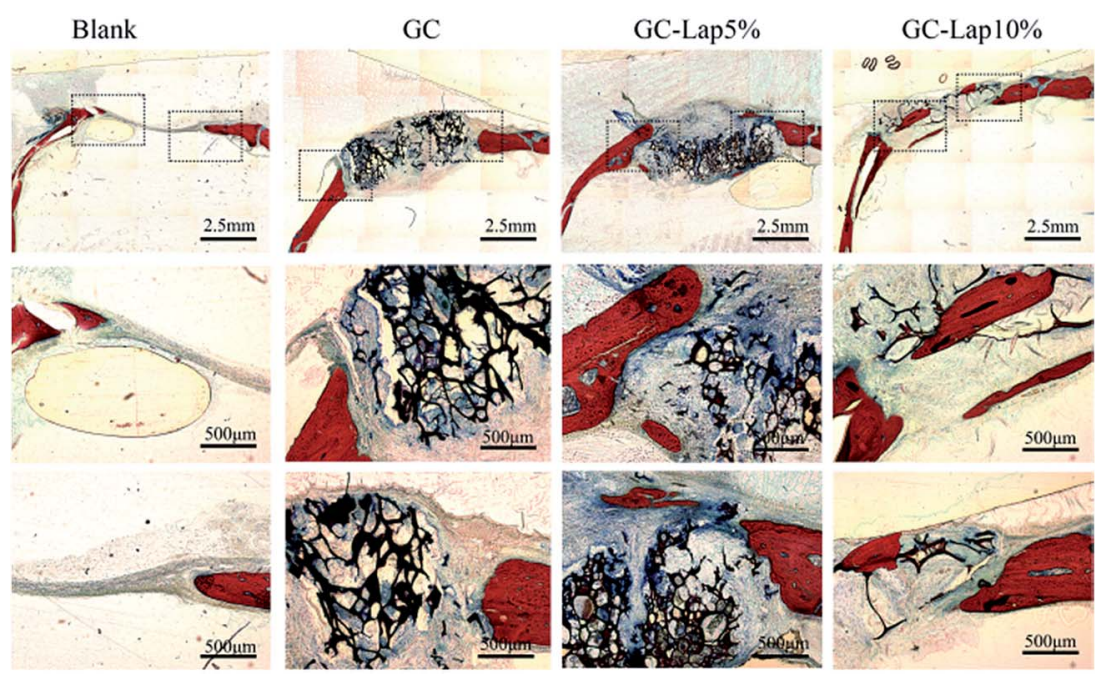

Fig. 13 VG staining of cranial bone for the pure GC, GC-Lap5\%, and GC-Lap10\% scaffolds at 8 weeks after implantation.

A light microscopic examination of van Gieson's picrofuchsin staining of newly formed bone at 8 weeks further supported the results of the micro-CT analysis (Fig. 13). The percentages of newly formed bone area at 8 week was higher in the defect of GC-Lap10\% $(28.05 \pm 2.01 \%)$ than that of GC-Lap5\% $(16.32 \pm 1.26 \%)$ and GC $(2.04 \pm$ $0.68 \%$ ). There was no evidence of new bone deposition in the GC scaffold group except at the border between the defect area and original bone, while in GC-Lap5\% group, only a small quantity of red-stained bone tissue was distributed in the defect area.

\section{Conclusions}

A novel composite scaffold was fabricated by incorporating Lap nanoclay into a gelatin/CMC matrix by freeze drying. Lap incorporation increased the mechanical properties of GC scaffolds while decreasing pore size, swelling, and degradation. A scaffold with $10 \mathrm{wt} \%$ Lap was ideal for promoting rBMSC attachment, proliferation, and osteogenic differentiation. The in vivo experiments revealed a gradual growth of bone tissue into the scaffolds. These results indicate that GCLap10\% scaffolds are a suitable material for BTE applications.

\section{Conflicts of interest}

There are no conflicts to declare.

\section{Acknowledgements}

The project was supported by the National Natural Science Foundation of China (Grant No. 81171707 and Grant No. 81572156), the National High Technology Research and Development Program of China (863 Program: 2015AA020308).

\section{References}

1 C. T. Laurencin, A. M. Ambrosio, M. D. Borden and J. A. Cooper Jr, Annu. Rev. Biomed. Eng., 1999, 1, 19.

2 K. J. Burg, S. Porter and J. F. Kellam, Biomaterials, 2000, 21, 2347.

3 R. Langer and J. P. Vacanti, Science, 1993, 260, 920.

4 T. Kokubo, H. M. Kim and M. Kawashita, Biomaterials, 2003, 24, 2161.

5 J. S. Mao, L. G. Zhao, Y. J. Yin and K. D. Yao, Biomaterials, 2003, 24, 1067.

6 A. Nandakumar, C. Cruz, A. Mentink, Z. Tahmasebi Birgani, L. Moroni, C. van Blitterswijk and P. Habibovic, Acta Biomater., 2013, 9, 5708.

7 J. Venkatesan, P. A. Vinodhini, P. N. Sudha and S. K. Kim, Adv. Food Nutr. Res., 2014, 73, 59.

8 S. Hammouche, D. Hammouche and M. McNicholas, Curr. Stem Cell Res. Ther., 2012, 7, 134.

9 R. Yunus Basha, T. S. Sampath Kumar and M. Doble, Mater. Sci. Eng., C, 2015, 57, 452.

10 H. Ehrlich, E. Steck, M. Ilan, M. Maldonado, G. Muricy, G. Bavestrello, Z. Kljajic, J. L. Carballo, S. Schiaparelli, A. Ereskovsky, P. Schupp, R. Born, H. Worch, V. V. Bazhenov, D. Kurek, V. Varlamov, D. Vyalikh, K. Kummer, V. V. Sivkov, S. L. Molodtsov, H. Meissner, G. Richter, S. Hunoldt, M. Kammer, S. Paasch, V. Krasokhin, G. Patzke, E. Brunner and W. Richter, Int. J. Biol. Macromol., 2010, 47, 141.

11 E. Landi, F. Valentini and A. Tampieri, Acta Biomater., 2008, 4, 1620.

12 V. K. Nandagiri, P. Gentile, V. Chiono, C. Tonda-Turo, A. Matsiko, Z. Ramtoola, F. M. Montevecchi and G. Ciardelli, J. Mech. Behav. Biomed. Mater., 2011, 4, 1318.

13 J. Li, Y. Chen, Y. Yin, F. Yao and K. Yao, Biomaterials, 2007, 28, 781 .

14 Q. Xing, K. Yates, C. Vogt, Z. Qian, M. C. Frost and F. Zhao, Sci. Rep., 2014, 16, 4706. 
15 D. Mishra, B. Bhunia, I. Banerjee, P. Datta, S. Dhara and T. K. Maiti, Mater. Sci. Eng., C, 2011, 31, 1295.

16 L. Upadhyaya, J. Singh, V. Agarwal and R. P. Tewari, J. Controlled Release, 2014, 186, 54.

17 L. Upadhyaya, J. Singh, V. Agarwal and R. P. Tewari, Carbohydr. Polym., 2013, 91, 452.

18 P. J. Schexnailder, A. K. Gaharwar, R. L. Bartlett 2nd, B. L. Seal and G. Schmidt, Macromol. Biosci., 2010, 10, 1416. 19 A. K. Gaharwar, V. Kishore, C. Rivera, W. Bullock, C. J. Wu, O. Akkus and G. Schmidt, Macromol. Biosci., 2012, 12, 779.

20 G. Ozkoc, S. Kemaloglu and M. Quaedflieg, Polym. Compos., 2010, 31, 674 .

21 H. M. Lewkowitz-Shpuntoff, M. C. Wen, A. Singh, N. Brenner, R. Gambino, N. Pernodet, R. Isseroff, M. Rafailovich and J. Sokolov, Biomaterials, 2009, 30, 8.

22 G. Nitya, G. T. Nair, U. Mony, K. P. Chennazhi and S. V. Nair, J. Mater. Sci.: Mater. Med., 2012, 23, 1749.

23 A. K. Gaharwar, P. J. Schexnailder, B. P. Kline and G. Schmidt, Acta Biomater., 2011, 7, 568.

24 A. K. Gaharwar, S. Mukundan, E. Karaca, A. DolatshahiPirouz, A. Patel, K. Rangarajan, S. M. Mihaila, G. Iviglia, H. Zhang and A. Khademhosseini, Tissue Eng., Part A, 2014, 20, 2088.

25 C. Wang, S. Wang, K. Li, Y. Ju, J. Li, Y. Zhang, J. Li, X. Liu, X. Shi and Q. Zhao, PLoS One, 2014, 23, e99585.

26 A. H. Ambre, D. R. Katti and K. S. Katti, J. Biomed. Mater. Res., Part A, 2013, 101, 26.

27 H. Zhuang, J. P. Zheng, H. Gao and K. De Yao, J. Mater. Sci.: Mater. Med., 2007, 18, 951.

28 A. K. Gaharwar, S. M. Mihaila, A. Swami, A. Patel, S. Sant, R. L. Reis, A. P. Marques, M. E. Gomes and A. Khademhosseini, Adv. Mater., 2013, 25, 3329.

29 S. M. Mihaila, A. K. Gaharwar, R. L. Reis, A. Khademhosseini, A. P. Marques and M. E. Gomes, Biomaterials, 2014, 35, 9087.

30 D. Su, L. Jiang, X. Chen, J. Dong and Z. Shao, ACS Appl. Mater. Interfaces, 2016, 8, 9619-9628.

31 S. J. Hollister, Nat. Mater., 2005, 4, 518-524.

32 J. Xie, C. Peng, Q. Zhao, X. Wang, H. Yuan, L. Yang, K. Li, X. Lou and Y. Zhang, Acta Biomater., 2016, 29, 365.

33 K. J. Livak and T. D. Schmittgen, Methods, 2001, 25, 402.

34 D. Zou, Z. Zhang, D. Ye, A. Tang, L. Deng, W. Han, J. Zhao,

S. Wang, W. Zhang, C. Zhu, J. Zhou, J. He, Y. Wang, F. Xu,

Y. Huang and X. Jiang, Stem Cells, 2011, 29, 1380.

35 Y. Takahashi and Y. Tabata, J. Biomater. Sci., Polym. Ed., 2004, 15, 41-57.

36 G. Akay, M. Birch and M. Bokhari, Biomaterials, 2004, 25, 3991.

37 X. Liu and P. X. Ma, Ann. Biomed. Eng., 2004, 32, 477.

38 K. Maji, S. Dasgupta, K. Pramanik and A. Bissoyi, Int. J. Biomater., 2016, 2016, 9825659.

39 S. Dhivya, S. Saravanan, T. P. Sastry and N. Selvamurugan, J. Nanobiotechnol., 2015, 13, 40.

40 J. Huang, S. M. Best, W. Bonfield, R. A. Brooks, N. Rushton, S. N. Jayasinghe and M. J. Edirisinghe, J. Mater. Sci.: Mater. Med., 2004, 15, 441.
41 S. Kim, M. E. Nimni, Z. Yang and B. Han, J. Biomed. Mater. Res., Part B, 2005, 75, 442.

42 C. Yang, L. Xu, Y. Zhou, X. M. Zhang, X. Huang, M. Wang, Y. Han, M. L. Zhai, S. C. Wei and J. Q. Li, Carbohydr. Polym., 2010, 82, 1297.

43 L. Ding, Y. Hu, Y. Luo, J. Zhu, Y. Wu, Z. Yu, X. Cao, C. Peng, X. Shi and R. Guo, Biomater. Sci., 2016, 4, 474.

44 K. C. Kavya, R. Jayakumar, S. Nair and K. P. Chennazhi, Int. J. Biol. Macromol., 2013, 59, 255.

45 C. Sharma, A. K. Dinda, P. D. Potdar, C. F. Chou and N. C. Mishra, Mater. Sci. Eng., C, 2016, 64, 416.

46 G. F. Perotti, H. S. Barud, Y. Messaddeq, S. J. L. Ribeiro and V. R. L. Constantino, Polymer, 2011, 52, 157.

47 C.-H. Lee, M. Kato and A. Usuki, J. Mater. Chem., 2011, 21, 6844.

48 S. Bose, M. Roy and A. Bandyopadhyay, Trends Biotechnol., 2012, 30, 546-554.

49 J. Ran, H. Zeng, J. L. Pathak, P. Jiang, Y. Bai, P. Yan, G. Sun, X. Shen, H. Tong and B. Shi, Biomacromolecules, 2017, 18, 3788-3801.

50 N. Pramanik, D. Mishra, I. Banerjee, T. Kumar Maiti, P. Bhargava and P. Pramanik, Int. J. Biomater., 2009, 512417.

51 G. Orive, R. M. Hernández, A. R. Gascón, M. Igartua and J. L. Pedraz, Int. J. Pharm., 2003, 259, 57.

52 S. Agacayak, B. Gulsun, M. C. Ucan, E. Karaoz and Y. Nergiz, Eur. Rev. Med. Pharmacol. Sci., 2012, 16, 679-686.

53 T. Gao, H. T. Aro, H. Ylänen and E. Vuorio, Biomaterials, 2001, 22, 1475.

54 I. D. Xynos, A. J. Edgar, L. D. K. Buttery, L. L. Hench and J. M. Polak, J. Biomed. Mater. Res., 2001, 55, 151.

55 H. Zreiqat, C. R. Howlett, A. Zannettino, P. Evans, G. SchulzeTanzil, C. Knabe and M. Shakibaei, J. Biomed. Mater. Res., 2002, 62, 175.

56 A. K. Gaharwar, C. Rivera, C.-J. Wu, B. K. Chan and G. Schmidt, Mater. Sci. Eng., C, 2013, 33, 1800.

57 D. W. Thompson and J. T. Butterworth, J. Colloid Interface Sci., 1992, 151, 236.

58 D. M. Reffitt, N. Ogston, R. Jugdaohsingh, H. F. J. Cheung, B. A. J. Evans, R. P. H. Thompson, J. J. Powell and G. N. Hampson, Bone, 2003, 32, 127.

59 H. Harada, S. Tagashira, M. Fujiwara, S. Ogawa, T. Katsumata, A. Yamaguchi, T. Komori and M. Nakatsuka, J. Biol. Chem., 1999, 274, 6972.

60 J. M. Oliveira, M. T. Rodrigues, S. S. Silva, P. B. Malafaya, M. E. Gomes, C. A. Viegas, I. R. Dias, J. T. Azevedo, J. F. Mano and R. L. Reis, Biomaterials, 2006, 27, 6123.

61 C. G. Bellows, J. E. Aubin and J. N. Heersche, Bone Miner., 1991, 14, 27.

62 A. J. Mieszawska, N. Fourligas, I. Georgakoudi, N. M. Ouhib, D. J. Belton, C. C. Perry and D. L. Kaplan, Biomaterials, 2010, 31, 8902.

63 J. H. Lee, N. G. Rim, H. S. Jung and H. Shin, Macromol. Biosci., 2010, 10, 173.

64 M. A. Saghiri, A. Asatourian, J. Orangi, C. M. Sorenson and N. Sheibani, Crit. Rev. Oncol. Hematol., 2015, 96, 143. 